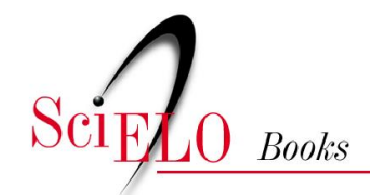

\title{
La vida del barrio y su influencia en el entorno alimentario
}

\author{
Jesús Rivera Navarro \\ María Sandín Vázquez \\ Marta Gutiérrez Sastre \\ Paloma Conde Espejo \\ Manuel Franco Tejero
}

\section{SciELO Books / SciELO Livros / SciELO Libros}

NAVARRO, JR., et al. La vida del barrio y su influencia en el entorno alimentario. In: PRADO, SD., et al. orgs. Estudos socioculturais em alimentação e saúde: saberes em rede. [online]. Rio de Janeiro: EDUERJ, 2016. Sabor metrópole series, vol. 5, pp. 103-123. ISBN: 978-85-7511-456-8. Available from: doi: 10.7476/9788575114568. Also available in ePUB from: http://books.scielo.org/id/37nz2/epub/prado-9788575114568.epub

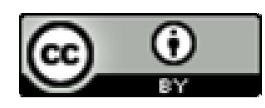

All the contents of this work, except where otherwise noted, is licensed under a Creative Commons Attribution 4.0 International license.

Todo o conteúdo deste trabalho, exceto quando houver ressalva, é publicado sob a licença Creative Commons Atribição 4.0.

Todo el contenido de esta obra, excepto donde se indique lo contrario, está bajo licencia de la licencia $\underline{\text { Creative Commons }}$ $\underline{\text { Reconocimento } 4.0 .}$. 


\section{La vida del barrio y su influencia en el entorno alimentario ${ }^{1}$}

Jesús Rivera Navarro

María Sandin Vázquez

Marta Gutiérrez Sastre

Paloma Conde Espejo

Manuel Franco Tejero

\section{Introducción}

Concebimos la alimentación no sólo como una forma de ingerir nutrientes (proteínas, vitaminas, minerales etc.) sino como un espacio simbólico en el cual se reproducen todas las dimensiones sociales existentes (Alonso, 2005); en este sentido, el concepto de "habitus" (conjunto de esquemas generativos a partir de los cuales los sujetos perciben el mundo y actúan en él) (Bourdieu, 1999) conecta con la idea de alimentación expresada anteriormente, pudiendo ayudar a explicar las diferencias de prácticas de alimentación entre unos grupos sociales y otros y también entre unos colectivos y otros (como colectivos de inmigrantes, grupos de edad etc.). Las prácticas de alimentación mencionadas han ido cambiando al mismo tiempo que lo han ido haciendo el sistema productivo, los modelos de consumo, las estructuras urbanas y los estilos de vida (López García y López

1 Los datos que se muestran en este capítulo de libro proceden del estudio piloto realizado, con metodología cualitativa, en el distrito municipal de Ciudad Lineal (Madrid, España). El marco de este estudio piloto fue el estudio Heart Healthy Hoods, proyecto financiado por Consejo Europeo de Investigación (Starting Grant, 2013) y cuyo investigador principal es el Dr. Manuel Franco Tejero. 
López, 2003), produciéndose una individualización y una fragmentación de las identidades, junto a una tendencia a la disolución de las redes y vínculos más sólidos (Sennett, 2000). Este es el contexto que debemos de tener en cuenta a la hora de analizar tanto los procesos alimentarios como sus entornos.

Actualmente, hay un gran número de estudios y publicaciones que constatan la influencia que tiene el entorno, como el barrio, en la dieta (Rahmanian et al., 2014). La gran mayoría de dichos estudios se basan en el análisis de la existencia de infraestructuras y recursos presentes en el barrio relacionados con la alimentación, como por ejemplo, el número y tipo de tiendas de alimentación, cafeterías, restaurantes, locales de comida rápida, y la distancia entre ellas, la disponibilidad de alimentos sanos, su precio etc., y su influencia en la dieta. Este tipo de investigaciones se caracterizan por utilizar técnicas cuantitativas que miden los recursos antedichos y su relación con la nutrición (Franco et al., 2009; Larson et al., 2009; Minaker et al., 2013; Morland et al., 2002; Pruchno et al., 2014). Otro común denominador muy frecuente en los trabajos que se ocupan del estudio de esta dimensión del entorno urbano es la consideración de las desigualdades socio-económicas en el examen de la relación entre los barrios, la alimentación y los problemas de salud derivadas de ésta, como la obesidad o la diabetes tipo II (Black et al., 2012; Bodicoat et al., 2014; Burdette e Whitaker, 2004; Caspi et al., 2012; Cetateanu e Jones, 2014; Drewnowski, 2009; Ford e Dzewaltowski, 2011; Keita et al., 2011; Münster et al., 2009; Smalls et al., 2014).

No podemos dejardeseñalarinvestigaciones másespecíficas que relacionan el entorno alimentario (fundamentalmente el acceso a establecimientos de comida sana o comida rápida) y otros factores, como el nivel socioeconómico (Brown e Brewster, 2015; Richardson et al., 2014), llegando a la conclusión de que a peor nivel socioeconómico existe una mayor probabilidad de vivir en un entorno alimentario menos favorecedor de la salud.

En España, las publicaciones existentes en torno a este tema han sido mucho menos profusas que en el ámbito anglosajón. El Estudio de Salud de la Ciudad de Madrid (Díaz, 2008) muestra una clara asociación entre la prevalencia de sobrepeso y/o obesidad y la clase social. La clase social más desfavorecida presenta cifras más elevadas para ambas condiciones: el riesgo de sobrepeso u obesidad aumenta discretamente en las clases más precarias y, controlando el efecto de sexo y edad, observamos que la probabilidad (Odds Ratio) de padecer exceso de peso en cualquier grado es 1,7 veces mayor en 
individuos de clase $\mathrm{V}$ (clase social más desfavorecida) respecto a los de clase I (Grupo de Trabajo de la SEE y de la SEMFC, 2000). ${ }^{2}$ La prevalencia de sobrepeso y obesidad presenta una relación inversa tanto con la clase social como con el nivel de renta de los barrios; de hecho, la prevalencia más elevada se produce en zonas de nivel socio-económico muy bajas, como Vicálvaro, Villaverde y Usera (53\%, 52\% y 49\% respectivamente), que contrasta con poblaciones de nivel más elevado en dónde la prevalencia es mucho más baja, como Salamanca, Centro, Chamberí y Chamartín (37\%, 35\%, 34\% y $32 \%$ respectivamente).

Otro estudio, realizado en España, y publicado en la revista Medicina Clínica, muestra también una clara relación entre el riesgo de obesidad y la clase social, basándose en la Encuesta Nacional de Salud de 2006 (OrtízMoncada et al., 2011).

Como indicamos anteriormente, la mayor parte de las investigaciones realizadas ha tenido una orientación cuantitativa, aunque no han faltado algunos trabajos que han basado su metodología en técnicas cualitativas, lo que ha permitido indagar, en profundidad, en aspectos que definen, en gran parte, la vida de barrio como la influencia de la cultura, los valores y las normas sociales que, a su vez, afectan a la nutrición y, por ende, a la salud (Kaufman e Karpati, 2007; Travers, 1996; Inglis, Ball e Crawford, 2005). Consideramos que dichos aspectos, que van a determinar el entorno alimentario, pueden llegar a ser igual o más importantes que el número de tiendas con productos saludables existentes en un determinado barrio o la disponibilidad de productos saludables o no saludables en dichos barrios. Sin embargo, este tipo de trabajos son relativamente escasos (en España por ejemplo no hay ninguno) y lo consideramos necesario para tratar de profundizar en factores determinantes de la salud en los barrios, como los

2 La clasificación de clases sociales, que se presenta en este trabajo, está basada en la Clasificación Nacional de Ocupaciones de 1994, cuya tipología abreviada es: I. Directivos de la Administración pública y de empresas de 10 o más asalariados. Profesiones asociadas a titulaciones de segundo y tercer ciclo universitario. II. Directivos de empresas con menos de 10 asalariados. Profesiones asociadas a una titulación de primer ciclo universitario. Técnicos. Artistas. Deportistas. III. Empleados de tipo de administrativo y profesionales de apoyo a la gestión administrativa y financiera. "Trabajadores de los servicios personales y de seguridad. Trabajadores por cuenta propia. Supervisores de trabajadores manuales. IVa. Trabajadores manuales cualificados. IVb. Trabajadores manuales semicualificados. V. Trabajadores no cualificados". 
valores, ${ }^{3}$ la tradición cultural, la percepción subjetiva del entorno y las normas sociales implícitas, así como los cambios acaecidos en dichas dimensiones. Dichos factores son muy complicados de captar de forma cuantitativa y ese es el principal motivo por el que hemos optado por abordar la relación entre el entorno alimentario y la estructura social del barrio a través de técnicas de investigación cualitativas, en este caso la entrevista semi-estructurada.

\section{Objetivos y metodología}

El objetivo principal de este estudio es analizar la relación entre el entorno alimentario y la estructura social del barrio, mediante la descripción de elementos clave en la vida de un barrio de nivel socioeconómico medio-bajo.

La investigación, que presentamos en este trabajo, está integrada en el proyecto "Heart Healthy Hoods" (ERC Starting Grant, 2013), que trata de analizar las desigualdades en salud existentes entre los diferentes diferentes barrios de Madrid (España) desde el estudio del entorno urbano y su relación con los factores de riesgo de enfermedades cardiovasculares. Los datos que mostramos forman parte del estudio piloto realizado en el marco del $\mathrm{HHH}$ Project, con metodología cualitativa. En el presente trabajo analizamos los datos relacionados con el ámbito alimentario, en concreto el análisis de las relaciones entre los vecinos y el entorno alimentario y cómo la crisis económica ha influido en dicha interacción.

Nuestro estudio tuvo una duración aproximada de 12 meses (de enero de 2014 a enero de 2015). En el ámbito de la metodología cualitativa, se utilizó la técnica de entrevista semi-estructurada, con el propósito de captar las percepciones que las personas elegidas, según los perfiles que se buscaban, tenían del barrio, de su transformación en los últimos años o décadas y su relación con las principales variables de la salud.

El estudio, que presentamos, se realizó en los barrios de Ventas, Pueblo Nuevo y Quintana, en la parte sur del distrito de Ciudad Lineal en el municipio de Madrid. Como otras muchas zonas urbanas que se desarrollaron

\footnotetext{
3 Nos referimos a los valores que pueden tener influencia en la dieta, como la importancia que se da a las relaciones de vecindad, a la consolidación de las redes sociales en el barrio o la percepción del barrio como eje central de la cotidianidad. De forma inversa, podríamos distinguir otro tipo de valores contrapuestos a los recientemente expuestos, como la "economización" del tiempo o la percepción del barrio como lugar-dormitorio.
} 
con el crecimiento demográfico y urbanístico de la capital española, estos barrios se originaron a partir de los años cincuenta del siglo XX tratando de dar respuesta a la fuerte demanda de vivienda de los grupos poblacionales procedentes del éxodo rural (Capel, 1997). La configuración urbanística de los barrios refleja ese momento, con un trazado viario desordenado, calles estrechas y empinadas y viviendas antiguas que apenas se han modernizado, buena parte de ellas sin ascensor ni calefacción central.

Con el auge económico de comienzos del XXI y la llegada de población inmigrante al país (Colectivo IOE, 2005), la zona experimentó un importante cambio poblacional. En 2006 se encontraba registrada en el catálogo de barrios vulnerables del ministerio de Fomento por contar con tasas de inmigración por encima del 20\%. Los efectos derivados de la crisis económica han propiciado el descenso generalizado del número de inmigrantes residentes, sin embargo el porcentaje de población extranjera que sigue residiendo en la zona se mantiene por encima de la media madrileña (13.1\%), especialmente en Quintana con un $16.7 \%$ pero también en Pueblo Nuevo (15.8\%) y Ventas (15.5\%). Respecto al envejecimiento, los tres barrios superan la media de la capital (20.2\%) ya que Ventas, Quintana y Pueblo Nuevo tienen un 25.1\%, un $22.0 \%$ y $23.8 \%$ de población mayor de 65 años, respectivamente. Un último dato para conocer la composición del barrio es el que tiene que ver con las tasas de paro registrado, que muestran un desempleo por encima del 15\% en los tres barrios, de nuevo por encima del $13.81 \%$ de media del municipio. La densidad de población, según el Padrón municipal del 2010, se sitúa muy por encima de la media en la capital madrileña $5.413 \mathrm{~h} / \mathrm{km}^{2}$ - con $16.419 \mathrm{~h} /$ $\mathrm{km}^{2}$ en Ventas, 27.815 en Pueblo Nuevo y 35.925 en Quintana.

Se realizaron 16 entrevistas a "informantes claves" en los barrios antes mencionados. Dichos “informantes claves” respondían a perfiles de la población del barrio que habíamos acordado previamente entre los investigadores, y los cuales nos podían suministrar información sobre factores y aspectos que podrían incidir en la salud de la población que vivía en el barrio. De una

\footnotetext{
4 El concepto de informantes claves procede del campo de la antropología, concretamente de los métodos etnográficos y, dentro de estos, de la técnica de observación participante. Se definen como aquellas personas que, por sus vivencias, capacidad de empatizar y relaciones que tienen en el campo pueden asesorar al investigador convirtiéndose en una fuente importante de información a la vez que le va abriendo el acceso a otras personas y a nuevos escenarios (Taylor e Bodgan, 1998).
} 
manera heterodoxa, y con el propósito de realizar un estudio piloto que nos alumbrará en el conocimiento del impacto del barrio y sus dimensiones en la salud en general, y en la salud cardiovascular en particular, utilizamos informantes claves para entrevistarles y extraer de sus discursos elementos que nos permitieran abordar de una manera convincente el análisis de otros barrios.

Los participantes respondían a perfiles específicos, que se detallan a continuación en la tabla 1 .

Tabla 1. Perfil de las personas entrevistadas

\begin{tabular}{|c|c|c|c|c|c|}
\hline Sexo & Edad (años) & Profesión & $\begin{array}{c}\text { Estado } \\
\text { civil }\end{array}$ & Procedencia & $\begin{array}{c}\text { Número de } \\
\text { entrevista }\end{array}$ \\
\hline Hombre & 48 & $\begin{array}{l}\text { Pequeño empresario } \\
\text { (regenta una carnicería) }\end{array}$ & Casado & Nativo & $1^{\mathrm{a}}$ \\
\hline Hombre & 85 & Jubilado & Casado & Nativo & $2^{a}$ \\
\hline Hombre & 63 & $\begin{array}{l}\text { Bedel del centro de sa- } \\
\text { lud "Daroca" }\end{array}$ & Soltero & Nativo & $3^{\mathrm{a}}$ \\
\hline Hombre & 62 & $\begin{array}{l}\text { Jubilado (activista en } \\
\text { una asociación de veci- } \\
\text { nos de Ciudad Lineal) }\end{array}$ & Soltero & Nativo & $4^{\mathrm{a}}$ \\
\hline Hombre & 42 & $\begin{array}{l}\text { Servicio de limpiezas. } \\
\text { Implicado en la asocia- } \\
\text { ción de vecinos y en la } \\
\text { vida del barrio }\end{array}$ & Soltero & Inmigrante & $5^{a}$ \\
\hline Hombre & 65 & Prejubilado & Casado & Nativo & $6^{\mathrm{a}}$ \\
\hline Hombre & 63 & $\begin{array}{l}\text { Concejal del PSOE } \\
\text { (Partido socialista Obre- } \\
\text { ro Español) en el distri- } \\
\text { to de Ciudad Lineal } \\
\end{array}$ & Casado & Nativo & $7^{a}$ \\
\hline Mujer & 83 & Jubilada & Viuda & Nativa & $8^{a}$ \\
\hline Mujer & 58 & Desempleada & Soltera & Nativa & $9^{\mathrm{a}}$ \\
\hline Mujer & 45 & Desempleada & Casada & Nativa & $10^{\mathrm{a}}$ \\
\hline Mujer & 69 & Jubilada & Casada & Nativa & $11^{\mathrm{a}}$ \\
\hline Mujer & 41 & $\begin{array}{l}\text { Profesora Colegio In- } \\
\text { fantil }\end{array}$ & No se sabe & Nativa & $12^{\mathrm{a}}$ \\
\hline Mujer & 53 & $\begin{array}{l}\text { Médica. Directora cen- } \\
\text { tro de salud Daroca }\end{array}$ & No se sabe & Nativa & $13^{\mathrm{a}}$ \\
\hline Mujer & 51 & $\begin{array}{l}\text { Médica. Directora Cen- } \\
\text { tro Madrid Salud Ciu- } \\
\text { dad Lineal }\end{array}$ & No se sabe & Nativa & $14^{\mathrm{a}}$ \\
\hline
\end{tabular}




\begin{tabular}{|c|c|c|c|c|c|}
\hline Mujer & 52 & $\begin{array}{l}\text { Coordinadora de Cen- } \\
\text { tros de Mayores de Ciu- } \\
\text { dad Lineal }\end{array}$ & No se sabe & Nativa & $15^{\mathrm{a}}$ \\
\hline Mujer & 41 & $\begin{array}{l}\text { Técnica en Animación } \\
\text { Sociocultural (ETA- } \\
\text { SOC). Centro "Manuel } \\
\text { Alexandre" }\end{array}$ & No se sabe & Nativa & $16^{\mathrm{a}}$ \\
\hline
\end{tabular}

Podríamos dividir los perfiles de las personas entrevistadas en dos grupos: 1) perfiles que se refieren a vecinos del barrio, con al menos diez años de antigüedad en el mismo, que nos ofrecieron, en las entrevistas, diferentes percepciones del mismo, por su diferente edad, por ser nativo o inmigrante, y por estar jubilado/a o estar en activo; 2) perfiles que se relacionan con profesionales que, por su trabajo, tenían una posición "privilegiada" para observar el barrio (a sus habitantes, las relaciones que establecen, los cambios que se producen etc.); por ello, hemos elegido a directores y/o trabajadores de centros de salud - tanto relacionados con la práctica clínica, como con la Promoción de la Salud -, directores de colegio, coordinadores y/ técnicos de centros de mayores.

La selección de las personas entrevistadas se basó en un muestreo intencional estratificado (Patton, 2002) que incluía los perfiles antes mencionados. La forma de contactar con los "vecinos del barrio" y otros informantes clave fue a través de la información que nos suministraron profesionales de la Salud Pública y de la Atención Primaria, que fueron necesarios para diseñar el estudio general Heart Healthy Hoods $(\mathrm{HHH})$, y de la asociación de vecinos La Merced del Barrio de Quintana. También empleamos la técnica bola de nieve para completar algunos de los perfiles (Teddlie e Yu, 2007).

El criterio de inclusión que se utilizó fue que los participantes en el estudio llevasen más de diez años viviendo o residiendo en el barrio y el criterio de exclusión fue que los participantes tuviesen dificultades de comunicación. Todas las personas entrevistadas participaron en este estudio voluntariamente y firmaron un consentimiento informado. La investigación general en la cual se inserta este estudio fue aprobada por el comité de ética en la investigación regional de la Comunidad de Madrid.

Se utilizó la técnica de entrevista semi-estructurada porque consideramos que era el instrumento adecuado para captar "relatos" en los cuáles se plasmarán todos los elementos del barrio que pudieran tener algún tipo de relación con la 
salud. Las entrevistas constaban de un guion previo que variaba según el tipo de persona que se iba a interpelar, aunque se componía de una base importante de preguntas que se repetían en todas las entrevistas (Tabla 2). En las entrevistas que llevamos a cabo se incluyeron preguntas sobre la composición socio-geográfica del barrio, la percepción individual de las características del entorno del barrio relacionadas con la alimentación, la actividad física, el alcohol y el tabaco, y las normas sociales de los vecinos acerca de estos factores de riesgo cardiovascular que afectan a su salud. En el presente trabajo, como indicamos anteriormente, presentamos solamente los resultados relacionados con el ámbito alimentario.

La duración de dichas entrevistas fue de entre 45 minutos y 1 hora; en el caso de los profesionales, se realizaban en los centros donde normalmente trabajaban los individuos y, en el caso de los vecinos, en sus domicilios; pretendíamos que hubiera un ambiente cálido y cómodo en el cual los interlocutores hablaran, sin cortapisas, de su visión del barrio.

Las preguntas que aparecían en el guion no tenían que formularse necesariamente, en muchas ocasiones, las personas entrevistadas abordaban los temas, previamente planificados, sin necesidad que el investigador formulara los temas o preguntas directamente. En otras ocasiones, los entrevistadores nos veíamos obligados a plantear las cu estiones directamente.

Las entrevistas fueron realizadas por tres investigadores, todos ellos autores de este artículo. Una de las autoras realizó ocho entrevistas, y los otros dos cuatro cada uno.

Tabla 2. Preguntas o temas comunes en el guion de las entrevistas

Descripción del barrio

Descripción del barrio y de la gente que habita en él

Tiempo de residencia

Delimitación geográfica del barrio

Cambios más importantes

Usos del barrio

Lugar dónde se trabaja, se compra, se realiza ocio

Tiempo que se pasa en el barrio

Preocupaciones de las personas que habitan en el barrio

Funcionamiento de los servicios de salud, de educación y los sociales en el barrio 
Salud relacionada con el barrio

Elementos del barrio que influyen en la salud

Infraestructuras (parques, polideportivos, bares, estancos, establecimientos de venta de comida, etc.)

Prácticas o estilos de vida relacionadas con la salud

Valores relacionados con la estructura social, la cultura y el género

Propuestas de mejora del barrio en relación de la salud (diagnóstico y pronóstico).

La saturación en las entrevistas se producía cuando todos los temas que estaban en el guion de los investigadores eran abordados por los entrevistados y no se aportara ningún información nueva, es decir, que se empezara a producir un punto de repetición del discurso (Quine, 1999).

Todas y cada una de las entrevistas fueron grabadas en audio, una vez firmado el consentimiento informado, y luego transcritas por personas cualificadas y expertas en este procedimiento. Los investigadores supervisaron dichas transcripciones (Rivera-Navarro et al., 2009). Las citas o verbatim que aparecen en este artículo son citas literales (citadas por el tipo de entrevista y el número de página transcrita, ejemplo, entrev 2, 5: entrevista 2, número 5).

En este estudio piloto se utilizó la hermenéutica interpretativa como marco teórico para analizar el discurso. Este paradigma tiene como elementos principales la consideración del contexto en el que se ubique el objeto del estudio y la particularidad del caso analizado (González Monteagudo, 2000), es decir se enfatiza tanto el papel de los factores sociales como la capacidad de los actores (los entrevistados) en dar significado a su realidad. En el mundo de la salud, este marco teórico también ha sido utilizado en diferentes investigaciones (Amezcua e Gálvez Toro, 2002).

Los primeros cuatro autores de este artículo realizaron varias lecturas sistemáticas de las transcripciones, siguiendo el criterio de la calidad de la triangulación del investigador (Golafshani, 2003) y de acuerdo a los pasos de análisis en progreso (Taylor, 1998). Los mencionados investigadores realizaron una lista de categorías y subcategorías, siguiendo el siguiente procedimiento (Hsieh e Shannon, 2005): 1) Categorización - las categorías tienen un alto nivel de abstracción y cada categoría puede incluir varios conceptos, por ejemplo, la categoría "ruptura de la homogeneidad social" incluye conceptos como " toda la vida" y "la confianza"; 2) Subcategorización - las subcategorías tienen un menor nivel de abstracción y sirven para explicar, de forma más profusa, las categorías, de esta forma, en la categoría señalada 
anteriormente (ruptura de la homogeneidad social) podríamos encontrar la subcategoría "diferencias intergeneracionales".

Las categorías no necesariamente tienen que coincidir con los temas y/o preguntas planteadas en el guion.

\section{Desarrollo}

El barrio está sometido a diferentes dinámicas que contribuyen a la transformación del mismo. Detrás de dichas dinámicas, identificamos cuatro grandes factores de transformación en el barrio que son:

1) “Rupturadela homogeneidadsocial". Nos referimos fundamentalmente a las prácticas cotidianas de la gente mayor, que sigue manteniendo una forma de vida muy marcada por relaciones sociales y actividades de ocio y consumo dentro del propio barrio. Estas prácticas tratan de salvaguardar las formas de vida tradicional que valoran la estabilidad y la confianza que da el tiempo. Desde este punto de vista se distingue entre vecinos veteranos - o que llevan muchos años viviendo en el barrio - y vecinos recientes.

... amigos de hace 50 años... normalmente nos vemos por la calle, nos saludamos, hablamos, sabemos ya los vecinos que tienen ganas de charlar un poquito y que están solas, y que necesitan desahogarse un poco, pues les preguntas “¿Qué tal te encuentras? ¿Cómo vas de esto?”... Y luego ya viene la $2^{a}$ generación de estos vecinos que te dan los buenos días y las buenas tardes, pero que no conectas más con ellos porque son parejas jóvenes o parejas monoparentales o... (Entrev 11, 4)

2) "Nuevos valores y formas de vida", relacionados con la falta del tiempo, la concentración de actividades, el estrés y la convivencia con nuevas culturas, relacionadas con la llegada de inmigrantes.

la vida que llevamos en general es desquiciada del todo, hay a gente que no le parece mal, más normalita y está más a gusto, pero yo creo que tenemos el estilo de vida desquiciado, sobre todo porque dado lo mal que está organizada la economía se vive para trabajar pero porque no tienes otra en realidad o no tienes otra opción. (Entrev 4, 5) 
3) "Precarización productiva y empobrecimiento generalizado", producido fundamentalmente por la crisis, los tipos de contratos de trabajo precarios y la incorporación de la mujer al mercado laboral sin posibilidad de conciliación.

Antes era un barrio más de funcionarios, de..., sin ser nada del otro mundo pero se creía más [...] sociológicamente de clase media [...], es de clases populares, pero no hasta ahora que sí, que se ha deteriorado un poco, no de las más necesitadas o así. (Entrev 4, 1)

\section{Ruptura de la homogeneidad social y entorno alimentario}

Todas las categorías analizadas (ruptura de la homogeneidad social; nuevos valores y empobrecimiento) están atravesadas por la dimensión "cohorte generacional", de esta forma, las personas mayores interiorizan unos valores, relacionados con la tradición y con las dinámicas existentes en las zonas urbanas de antaño, que condicionan el acceso a determinados establecimientos y la forma de alimentarse. Esta forma de consumir y comprar productos alimenticios es diferente tanto respecto a las personas nativas más jóvenes, como a las personas inmigrantes.

En el factor "ruptura de la homogeneidad social", y como reacción hacia dicho factor, encontramos el concepto "de toda la vida". Los vecinos, que llevan más de quince años viviendo en el barrio, compran en tiendas "de toda la vida", es decir en tiendas que llevan mucho tiempo ubicadas en el barrio y cuyos trabajadores y/o propietarios son conocidos, desde hace mucho años, por las personas del entorno. Este concepto "de toda la vida" hace que los residentes, que llevan habitando más años en el barrio, compren en dichas tiendas "de toda la vida", basándose en argumentos como la confianza. Esta confianza, basada en aspectos afectivos y emocionales vinculadas a las personas que atienden al público, se traduce en una mayor percepción de seguridad a la hora de comprar los productos que se consideran adecuados. Es decir, hemos detectado la posible existencia de una norma que hace que, a pesar de tener mucha oferta de comercios para alimentación, la dieta sea más o menos variada en función del acceso a las tiendas "de toda la vida" (porque los vecinos "antiguos", que llevan más tiempo en el barrio, no compran en tiendas nuevas o en grandes establecimientos, al menos habitualmente). 
... La carne la compro aquí en eso, de toda la vida ya ... dan buena carne. (Entrev 2, 7)

Voy al de La Elipa que ya conozco a la gente... De toda la vida, claro, yo al carnicero ya le compro hace 30 años. (Entrev 6, 20)

Llevo 40 y tantos años con ellos, son paisanos míos, o sea que no... Otras veces me dice que no, y que no. $\mathrm{O}$ yo le digo "necesito una leche especial que necesito de lactosa o no sé qué", y él me la trae. Yo es que ya llevo 40 años comprando en ellos. Por eso digo yo "mis tiendas de barrio yo las reivindico"... yo reivindico las tiendas del barrio, porque la amistad que vas cogiendo... (Entrev 11, 10)

Por lo tanto, la "confianza" depositada en el establecimiento, y en la persona que normalmente atiende en dicha tienda, se convierte en una de las razones principales de la utilización de las tiendas de "toda la vida". Esta "confianza" no sólo consiste en sentirse cómodo en el interior del comercio, y en la existencia de una relación cordial con los dependientes, sino en que se produzca también una iniciativa, por parte de los vendedores, para recomendar productos alimenticios que puedan convenir a los vecinos, por su mayor calidad y por su mejor precio.

Confianza con las tiendas y, bueno, pues... Sí, sí, sí, muy importante. Para mí es importante porque por ejemplo en la pescadería no los conozco, que han venido muy jóvenes, han venido hace poco, y voy y compro, y compro bien porque compro lo que yo quiero y lo que conozco el género, pero no tengo la confianza esa de que ellos me digan: "oye, ¿Por qué no te llevas esto hoy?". (Entrev 8, 15)

El trato directo con el tendero, el vecino de enfrente, que te da la calidad del producto. (Entrev 14, 8)

Nuestros interlocutores más mayores explicitan que la confianza, y la querencia por las tiendas de "toda la vida", está determinada por la edad y por la cohorte generacional y, a su vez, por los estilos y formas de vida; de esta forma, se reconoce que el comportamiento, como consumidores, de las 
personas más jóvenes es diferente, en el sentido de la existencia de un mayor pragmatismo a la hora de comprar determinado, a su vez, por un uso distinto del tiempo.

Los chicos jóvenes no..., si trabaja el matrimonio tienen a lo mejor un día para hacer la compra, que puede ser el sábado o el domingo, entonces ellos se desplazan muchísimo mejor a un centro comercial donde pueden comprar de todo ..., las cajeras no la conocen, a no ser que haya pasado algún episodio o que tengas amistad o lo que sea, y sin embargo yo entro en mi súper y soy una de la familia. (Entrev 11, 12)

La distancia física, el poder ir andando desde el domicilio, al establecimiento, y la comodidad que ello proporciona, es otra ventaja añadida que potencia el uso de las tiendas "de toda la vida" o los supermercados más pequeños frente a los de grandes superficies.

No, yo no voy a comprar nunca (a los centros comerciales) porque me pilla lejos. Habitualmente compramos más cerca, que es como te digo en Día, que en Marcelino Álvarez hay un Día o en Supersol que está en la calle Alcalá, aquí en el Carmen, o en el Eroski que está en Virgen de Sagrario, no digo, en Hipercor que está en Virgen de Sagrario. (Entrev 6, 10)

En relación con esta cercanía de las tiendas al domicilio, existe preocupación, especialmente por parte de la gente mayor, de que cierren las tiendas que tienen más próximas (ya que les resulta más cómodo comprar allí). Además influye la accesibilidad (la inexistencia de barreras arquitectónicas) a las tiendas, que determina la comodidad para comprar.

A todas (las vecinas) les digo: "no dejéis de comprar que es la única tienda que tenemos en el barrio, no dejéis de comprar, vaya a ser la que tengan que cerrar porque no se compra”. (Entrev 8,10$)$

Lo que sí hicieron hace unos años, como este barrio tiene todo muchas cuestas, si subes de La Elipa para acá esto es como una meseta, es todo cuesta abajo. Entonces había un mercado, muy pequeñito..., pero no tenía casi acceso, porque son unas escaleras que no hay cristiano que las suba, bajarlas 
a lo mejor las bajabas, ipero subirlas una vez cargado! Entonces han hecho como unas rampas, que no sabes cuándo acaban, pero ya es accesible para bajar a Bami si bajas con el carrito de los niños o algo, ya te digo que tardas... (Entrev 11, 13)

Otro atributo ventajoso que se arroga a las tiendas "de toda la vida", y a los supermercados más pequeños, es la "variedad" de productos en dichos establecimientos, que hace más cómoda la compra, especialmente a la gente mayor (acudiendo una sola vez a la tienda, pueden comprar todo tipo de productos). Esta característica no es exclusiva de este tipo de tiendas, de hecho en los establecimientos más grandes, por su naturaleza, suelen tener más diversidad de productos, sin embargo, es una condición importante para la utilización de estas tiendas por parte de las personas nativas mayores del barrio.

Antes había un supermercado ahí que era más caro, que está cerrado ahora... y abrieron Ahorra Más y ahí es donde va mi señora, porque está ahí todo. (Entrev 2, 11)

Lo que más cerca te pilla y además que hay buen precio y hay mucho surtido para elegir y hay buena mercancía. (Entrev 6,10$)$

La dicotomía tiendas "de toda la vida" versus "grandes superficies o supermercados" es rota por la aparición, relativamente reciente, de tiendas regentadas por inmigrantes (tiendas pequeñas, en muchas ocasiones especializadas en géneros alimenticios como la fruta), que son vistas como una amenaza por los propietarios de tiendas "de toda la vida", aunque no parece, por las entrevistas realizadas, que sean visitadas por las personas nativas que viven en el barrio, al menos por las personas mayores. La amenaza referida se refleja en argumentos relativos a la "cercanía" de estas tiendas a los domicilios y al precio de los productos (se dice que son más baratos) que podría provocar que las personas mayores compren allí y no acudan a las tiendas de "toda la vida". Esto se vislumbra como un problema, puesto que se considera que en estas tiendas se venden productos de peor calidad (como veremos más adelante). 
Entonces una persona mayor yo reconozco que por qué va a ir hasta el mercado teniendo una frutería debajo de casa. ¿Es mejor el producto, es peor? Yo no voy a entrar en eso, no sé si es mejor o es peor, el trato yo creo que sí es distinto. (Entrev 1, 14)

\section{Inmigración, nuevos valores, estilos de vida y entorno alimentario}

Por otro lado, otro elemento clave que influye en la relación de los vecinos con el entorno alimentario es la inmigración. Se denota desconfianza hacia los comercios de "dueños extranjeros o inmigrantes", así como a los productos que allí se venden. Se consideran de peor calidad y de un origen dudoso, aun así el relativo éxito que tienen este tipo de tiendas se atribuyen a su flexibilidad horaria, mucho mayor que las tiendas "de toda la vida" o que otro tipo de establecimientos. Sin embargo, se considera que la forma de trato de los dependientes hacia el cliente es peor en las tiendas de los inmigrantes que en las "de toda la vida".

Esas son de extranjeros, de estos, escoges la fruta, [...] y lo coges y luego cuando llegas a casa y lo coges está pasado por dentro y todo. (Entrev 2, 14)

Es que las abren los domingos, los sábados y los domingos, es que eso es... ¿tú harías abrir una tienda todos los días, de 9 de mañana a 12 de la noche? (Entrev 3, 11)

Las mujeres inmigrantes han encontrado un nicho de mercado laboral relacionado con el trabajo doméstico, especialmente con el cuidado a las personas mayores. Este nuevo rol de cuidadoras ha producido que las mujeres inmigrantes se erijan en las protagonistas a la hora de variar la dieta de las personas que están cuidando (ya que cocinan según sus países de origen). Esta variación de la alimentación podría provocar no sólo consecuencias e impactos en la salud, sino también una mayor utilización de los establecimientos administrados por inmigrantes

[...] Muchas de las cuidadoras de nuestros ancianos son inmigrantes que les hacen sus comidas y las comidas latinoamericanas son muy ricas en hidratos de carbono y en grasas, pero es que las del Este ya son de morirte. (Entrev 13, 9) 


\section{El efecto de la crisis económica y el entorno alimentario}

Hay un elemento que aparece, de forma recurrente, en todas las entrevistas realizadas, que es la "crisis económica". Debemos tener en cuenta que cuando realizamos las entrevistas, el impacto de dicha crisis llevaba ya operando en España más de cuatros años y sus consecuencias se dejaban sentir en muchos estratos sociales de la población. Desde este punto de vista, el poder adquisitivo de los vecinos (no tanto el nivel socioeconómico del barrio) condiciona tanto la alimentación como el acceso a entornos alimentarios concretos. En el caso de las personas mayores, hay ocasiones que tienen que mantener a sus familias con las pensiones, lo que condiciona la calidad, variedad y cantidad de lo que compran y en dónde lo pueden adquirir. Es decir, el efecto de la crisis económica ha hecho cambiar patrones de alimentación en el barrio (hay abuelos que mantienen a hijos y nietos, y que se alimentan peor por ello).

Yo tengo hamburguesa porque tengo a mi nieto a comer y entonces tengo no sé cuánto... 3 o 4 euros... entonces o comemos hamburguesa o no comemos. (Entrev 13, 9)

La "crisis económica" condiciona el acceso a los entornos alimentarios, de esta forma, los vecinos identifican que los precios determinan los alimentos que se compran y dónde se compran y, por lo tanto, la búsqueda de los lugares más económicos y de las ofertas es una constante en algunas de nuestras entrevistas. En este caso, las limitaciones económicas hacen que varíe la norma social de uso de tiendas de toda la vida, primando la búsqueda de precios más baratos.

Yo voy a comprar el pescado allí porque es más barato. (Entrev 3,12)

[...] Si las ofertas son muy importantes, pues te coges el autobús que va para Vicálvaro y te deja en Carrefús... También voy al Carrefour, pero muy poco. Ya cuando me veo las ofertas que me interesan... (Entrev 11, 11) 
La "crisis económica" también condiciona "la manera de comprar", en el sentido que se compra menos cantidad y con los productos que se necesitan "dia a dia", sin acumular productos.

Se mueven mucho por el barrio, porque además piensa que son personas que tienen un nivel adquisitivo muy bajo y entonces van al DIA, entonces en la tienda que tienen más cercana pues es donde...si necesito una patata pues compro una patata, no compro cinco kilos. Me explico, ¿̨no? Entonces a nivel de comprar pues comprar en las tiendas del barrio. (Entrev 12, 14)

La "crisis económica" ha llevado a reducir la "calidad" de los productos que se compran, aunque dicha reducción se atribuye a ciertos sectores de población, los más depauperados, identificados con la población inmigrante; así mismo, se considera que los productos más baratos, relacionados con una peor calidad, se venden en tiendas de inmigrantes (ni en las de "toda la vida", ni en los supermercados grandes o pequeños). Por lo tanto, podríamos hablar de una identificación de calidad-precio-tipo de tienda.

Que no, que a mí esa fruta no, es que qué diferencia, que no es lo mismo una naranja que la compres ahí (en las tiendas de inmigrantes), y no son tan baratas tampoco, pero bueno. Eso sí, la pobre gente por desgracia sí tiene que comprar ahí. (Entrev 2, 13)

La "crisis económica" ha provocado que los mayores afectados por dicha crisis (los desempleados) se agrupen en organizaciones sin ánimo de lucro y consigan que ciertos negocios relacionados con la alimentación (como algunas panaderías) proporcionen pan gratis a las personas sin empleo. Podríamos considerar que, de alguna manera, se cambian las dinámicas mercantiles en relación a la alimentación por la crisis.

Hay una panadería aquí en la zona que ya vamos a empezar a funcionar en breve, nada más que haya unos pequeños toques que hay que matizar, que hemos conseguido que colaboren con el grupo, con este grupo de parados y un día a la semana va a haber pan gratis para los parados. (Entrev 10,5) 


\section{Conclusiones}

Los barrios, en una ciudad como Madrid, han estado sometidos a una serie de cambios, en las últimas décadas, que se han plasmado en una serie de tendencias como:

1. la ruptura de la homogeneidad social, en el sentido de que en los barrios hay mucha más diversidad cultural, reflejado en la existencia de colectivos inmigrantes, $y$ un mayor abanico en las percepciones de la realidad, reflejados en los jóvenes;

2. nuevos valores, relacionados con estilos de vida diferentes a los tradicionales, vinculados a un ritmo más acelerado en la cotidianidad, menos dedicación a la familia y más al trabajo (Artazcoz et al., 2006), pero también a llegada de inmigrantes con una visión del mundo diferente (Torres Pérez, 2005);

3. Precarización productiva y empobrecimiento generalizado de la población en las grandes urbes, vinculado, en gran medida, a la crisis económica;

Estas tendencias influyen tanto en el entorno alimentario como en el uso que se hace de los alimentos.

Las cohortes generacionales determinan la interiorización de valores que, a su vez, condicionan el uso de espacios relacionados con la venta de la alimentos y con la forma de alimentarse. La reacción ante la ruptura de la homogeneidad social en el ámbito del entorno alimentario la representa las tiendas de toda la vida. Este concepto se refiere a pequeños establecimientos, regentados por personas nativas, en muchos casos del mismo barrio, y en los cuales se venden productos relacionados con la alimentación como fruta, embutidos, conservas, etc. (no se suelen vender en estos comercios ni carne del día ni pescado fresco). Las personas que frecuentan este tipo de tiendas son personas que llevan mucho tiempo en el barrio, en muchos casos personas mayores. Las tiendas de toda la vida están asociadas a la confianza en lo que se compra, a la comodidad de su utilización, a su accesibilidad y a la pertenencia al barrio.

La dicotomía tiendas de toda la vida versus grandes superficies es fracturada por la irrupción, relativamente reciente, de tiendas gestionados por inmigrantes que genera resquemor y desconfianza entre las personas nativas (especialmente 
las mayores). Esta proliferación de las tiendas de los inmigrantes es favorecida por las cuidadoras inmigrantes de ancianos/as que tienen cierta autonomía para decidir la dieta de los receptores de atención (Vega Solis, 2009).

La crisis económica es un determinante fundamental en el uso de los entornos alimentarios, de tal forma que obligan en algunos casos a romper la querencia exclusiva de las "tiendas de toda la vida" y a buscar una mayor diversidad de los lugares en los que se compra, en función, fundamentalmente, de los precios. Además, dicha crisis condiciona también la forma de consumir y la calidad de los productos, además de provocar dinámicas solidarias y comunitarias en un entorno determinado por "la ley del mercado".

Este trabajo apunta a que la existencia de una serie de dinámicas, que dan explicación a la transformación social en el ámbito urbano, podrían explicar tanto el diferente uso del entorno alimentario, como la adopción de dietas concretas. Somos conscientes de las limitaciones de esta investigación, principalmente por su carácter de estudio piloto que no ha permitido la selección de una muestra estructural más adecuada. Sin embargo, creemos que estos resultados constituyen un primer paso para una mayor profundización en las categorías apuntadas y analizadas en este manuscrito.

\section{Referencias}

ALONSO, L. E. "Mitologías alimentarías cotidianas. Una relectura de Roland Barthes". Revista Internacional de Sociología (RIS), n. 40, 2005, pp. 79-107.

AMEZCUA, M. et al. "Los modos de análisis en investigación cualitativa en salud: perspectiva crítica y reflexiones en voz alta". Revista Española de Salud Pública, v. 76, n. 5, 2002, pp. 423-36.

ARTAZCOZ, L. et al. "El estrés en una sociedad instalada en el cambio". Gaceta Sanitaria, v. 20, n. 1, 2006, pp. 71-8.

BLACK, C. et al. "Variety and quality of healthy foods differ according to neighbourhood deprivation". Health \& Place, v. 18, n. 6, 2012, pp. 1.292-9.

BODICOAT, D. H. et al. "The association between neighbourhood greenspace and type 2 diabetes in a large cross-sectional study", British Medical Journal Open, v. 4, n. 12, 2014.

BOURDIEU, P. La distinción: criterios y bases sociales del gusto. Barcelona: Tauru, 1999.

BROWN, D. R. e BREWSTER, L. G. "The food environment is a complex social network". Social Science and Medicine, 2015. (mimeo)

BURDETTE, H. L. e WHITAKER, R. C. "Neighborhood playgrounds, fast food restaurants, and crime: relationships to overweight in low-income preschool children". Preventive Medicine, v. 38, n. 1, 2004, pp. 57-63. 
CAPEL, H. S. "Los inmigrantes en la ciudad. Crecimiento económico, innovación y conflicto social”. Scripta Nova: Revista Electrónica de Geografía y Ciencias Sociales, n. 1, 1997, pp. 1-13.

CASPI, C. E. et al. "The relationship between diet and perceived and objective access to supermarkets among low-income housing residents", Social Science and Medicine, v. 75, n. 7, 2012, pp. 1.254-62.

CETATEANU, A. e JONES, A. "Understanding the relationship between food environments, deprivation and childhood overweight and obesity: evidence from a cross sectional England-wide study”. Health \& Place, v. 27, 2014, pp. 68-76.

COLECTIVO IOE. “Inmigrantes extranjeros en España, ¿̇reconfigurando la sociedad?”. Panorama social, v. 1, 2005, pp. 32-47.

DÍAZ J. M. e ESTEBAN M. "Estudio de Salud de la Ciudad de Madrid". Madri, 2008. Instituto de Salud Pública, Madrid Salud. Disponível em: http://www.madridsalud.es/ publicaciones/OtrasPublicaciones/EstudioSaludCiudadMadrid.pdf.

DREWNOWSKI, A. "Obesity, diets and social inequalities". Nutrition Reviews, v. 67, sup. 1, 2009, pp. 36-9.

FORD, P. B. e DZEWALTOWSKI, D. A. "Neighborhood deprivation, supermarket availability, and BMI in low-income women: a multilevel analysis". Journal of Community Health, v. 36, n. 5, 2011, pp. 785-96.

FRANCO, M. et al. "Availability of healthy foods and dietary patterns: the Multi-Ethnic Study of Atherosclerosis". The American Journal of Clinical Nutrition, v. 89, n. 3, 2009, pp. 897-904.

GOLAFSHANI, N. "Understanding reliability and validity in qualitative research". The Qualitative Report, v. 8, n. 4, 2003, pp. 597-607.

GONZÁLEZ MONTEAGUDO, J. “El paradigma interpretativo en la investigación social y educativo: nuevas respuestas para viejos interrogantes”. Disponível em: http://institucional. us.es/revistas/cuestiones/15/art_16.pdf. Acesso em: 9 mar. 2015.

GRUPO de trabajo de la Sociedad Española de Epidemiología (SEE) y de la Sociedad Española de Medicina de Familia y Comunitaria (SEMFC). "Una propuesta de medida de la clase social”, Atención Primaria, v. 25, n. 5, 2000, pp. 350-63.

HHH. "The Heart Healthy Hoods Project: a multifaceted approach to cardiovascular diseases in European Cities". Disponível em: http://hhhproject.eu/.

HSIEH, H-F. e SHANNON, S. E. “Three approaches to qualitative content analysis". Qualitative Health Research, v. 15, n. 9, 2005, pp. 1.277-88.

INGLIS, V. et al. "Why do women of low socioeconomic status have poorer dietary behaviours than women of higher socioeconomic status? A qualitative exploration". Appetite, v. 45, n. 3, 2005, pp. 334-43.

KAUFMAN, L. e KARPATI, A. "Understanding the sociocultural roots of childhood obesity: food practices among Latino families of Bushwick, Brooklyn”. Social Science and Medicine, v. 64 , n. 11,2007 , pp. 2.177-88.

KEITA, A. D. et al. "Neighborhood perceptions affect dietary behaviors and diet quality". Journal of Nutrition Education and Behavior, v. 23, n. 2, 2011, pp. 183-96.

LARSON, N. I. et al. "Neighborhood environments: disparities in access to healthy foods in the U.S.”. American Journal of Preventive Medicine, v. 36, n. 1, 2009, pp. 74-81. 
LÓPEZ GARCÍA, D. e LÓPEZ LÓPEZ, J. A. Con la comida no se juega: alternativas autogestionarias a la globalización capitalista desde la agroecología y el consumo. Madri: Traficantes de Sueños, 2003.

MINAKER, L. M. et al. "Objective food environments and health outcomes". American Journal of Preventive Medicine, v. 45, n. 3, 2013, pp. 289-96.

MORLAND, K. et al. "Neighborhood characteristics associated with the location of food stores and food service places". American Journal of Preventive Medicine, v. 22, n. 1, 2002, pp. 23-9.

MÜNSTER, E. et al. "Over-indebtedness as a marker of socioeconomic status and its association with obesity: a cross-sectional study". BMC Public Health, v. 9, n. 286, 2009.

ORTÍZ-MONCADA, R. et al."Determinantes sociales de sobrepeso y obesidad en España 2006”, Medicina Clínica, v. 137, n. 15, 2011, pp. 678-84.

PATTON, M. Q. "Qualitative Research \& Evaluation Methods". SAGE Publications, 2002.

PRUCHNO, R. et al. "Neighborhood food environment and obesity in community-dwelling older adults: individual and neighborhood effects". American Journal of Public Health, v. 104, n. 5, 2014, pp. 924-29.

QUINE, S. "Focus groups". In KERR, C.B. et al. (orgs.). Handbook of Public Health methods. 3 ed. Londres: Sage Publications, 1999, pp. 527-32.

RAHMANIAN, E. et al. "The association between the built environment and dietary intake-a systematic review”. Asia Pacific Journal of Clinical Nutrition, v. 23, n. 2, 2014, pp. 183-96.

RICHARDSON, A. S. et al. "Neighborhood socioeconomic status and food environment: a 20 year longitudinal latent class analysis among CARDIA participants". Health \& Place, v. 30, 2014, pp. 145-53.

RIVERA-NAVARRO, J. et al. “The diagnosis of Tourette's Syndrome: communication and impact”. Clinical Child Psychology and Psychiatry, v. 14, n. 1, 2009, pp. 13-23.

SENETT, R. La corrosión del carácter: las consecuencias personales del trabajo en el nuevo capitalismo. Barcelona: Anagrama, 2009.

SMALLS, B. L. et al. "Effect of neighborhood factors on diabetes self-care behaviors in adults with type 2 diabetes". Diabetes Research and Clinical Practice, v. 106, n. 3, 2014, pp. 435-42.

TAYLOR, S. J. e BOGDAN, R. Introduction to Qualitative Research method. 3 ed. Nova Iorque: John Wiley \& Sons, 1998.

TEDDLIE, C. H. e YU, F. "Methods sampling. Typology with examples". Journal of Mixed Methods Research, v. 1, n. 1, 2007, pp. 77-100.

TORRES PÉREZ, F. "Los espacios públicos en la ciudad multicultural. Reflexiones sobre dos parques de Valencia”. Pensamiento crítico. Disponível em: www.pensamientocritico. org/frantorr20605.htm. Acesso em: 20 abr. 2015.

TRAVERS, K. D. “The social organization of nutritional inequities”. Social Science \& Medicine, v. 43, n. 4, 1996, pp. 543-53.

VEGA SOLIS, C. Culturas del cuidado en transición: espacios, sujetos e imaginarios en una sociedad de migración. Barcelona: Editorial UOC, 2009.

ZICK, C. et al. "Running to the store? The relationship between neighborhood environments and the risk of obesity". Social Science and Medicine, v. 69, n. 10, 2009, pp. 1.493-500. 